REIHE COMPUTATIONAL INTELLIGENCE SONDERFORSCHUNGSBEREICH 531

Design und Management komplexer technischer Prozesse und Systeme mit Methoden der Computational Intelligence

On The Effect of Populations in Evolutionary Multi-objective Optimization

Oliver Giel and Per Kristian Lehre

Nr. Cl-202/06

Interner Bericht

ISSN 1433-3325

Februar 2006

Sekretariat des SFB 531 . Universität Dortmund . Fachbereich Informatik/XI 44221 Dortmund · Germany

Diese Arbeit ist im Sonderforschungsbereich 531, „Computational Intelligence“, der Universität Dortmund entstanden und wurde auf seine Veranlassung unter Verwendung der ihm von der Deutschen Forschungsgemeinschaft zur Verfügung gestellten Mittel gedruckt. 


\title{
On The Effect of Populations in Evolutionary Multi-objective Optimization
}

\author{
Oliver Giel ${ }^{1 \star}$ and Per Kristian Lehre ${ }^{2 \star \star}$ \\ 1 Fachbereich Informatik, Lehrstuhl 2 \\ Universität Dortmund \\ 44221 Dortmund, Germany \\ oliver.giel@cs. uni-dortmund.de \\ 2 Dept. of Computer and Information Science \\ Norwegian University of Science and Technology \\ 7491 Trondheim, Norway \\ lehre@idi.ntnu.no
}

\begin{abstract}
Multi-objective evolutionary algorithms (MOEAs) have become increasingly popular as multi-objective problem solving techniques. Most studies of MOEAs are empirical. Only recently, a few theoretical results have appeared. It is acknowledged that more theoretical research is needed. An important open problem is to understand the role of populations in MOEAs. We present a simple bi-objective problem which emphasizes when populations are needed. Rigorous runtime analysis point out an exponential runtime gap between a population-based algorithm (SEMO) and several single individual-based algorithms on this problem. This means that among the algorithms considered, only the populationbased MOEA is successful and all other algorithms fail.
\end{abstract}

\section{Introduction}

The understanding of the role of populations in single-objective EAs has been supported by theoretical results $[11,12]$. For example, there are problems where reducing the parent population size by one leads to an exponential increase in the runtime [10].

In a population of a single-objective EA, all individuals are comparable. This is typically not the case for MOEAs. Hence, it is not obvious that results for single-objective problems carry over to MOEAs when applied to a truly multi-objective problem. The use of populations in multi-objective EAs is often motivated by the need to find a set of solutions (the Pareto set) rather than a single optimal solution. However, it is unclear whether one can achieve the same goal by restarting a single individual algorithm. Laumanns et al. [8] prove that some simple population based MOEAs can slightly outperform a single

\footnotetext{
* Supported by the German Research Foundation (DFG) as a part of the collaborative research center "Computational Intelligence" (SFB 531).

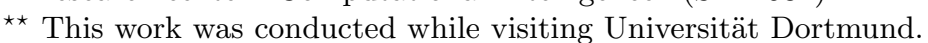


individual based approach called $\varepsilon$-constrained method. This result alone is not sufficient to understand the role of populations in MOEAs since the runtime gap is rather small, and it gives only little insight into why the population based approach can be superior. In this paper, we present a problem where many single individual-based algorithms, including the $\varepsilon$-constrained method, fail dramatically. Furthermore, the presented problem has a structure that can better explain why the individual based algorithms fail.

This paper is organized as follows. We introduce the MOEAs considered and the necessary notation in the next section. The objective function will be presented in Section 3. In Sections 4-6, we show that all considered single individual algorithms fail on this objective function. Finally, in Section 7, we prove that SEMO efficiently discovers all Pareto optimal points in the objective space.

\section{Preliminaries}

\subsection{Notation}

We assume that the reader is familiar with the concepts of multi-objective optimization (see, e.g., [2]). We consider a maximization problem $f:\{0,1\}^{n} \rightarrow \mathbb{R}^{m}$. For clarity, we say that a vector $b$ from the objective space $\mathbb{R}^{m}$ weakly dominates another vector $a$, denoted $a \preceq b$, if $a_{i} \leq b_{i}$, for all $i$. We say $b$ dominates $a$, denoted $a \prec b$, if $a \preceq b$ and $a_{i}<b_{i}$ for at least one index $i$. This notation will also be used for solutions $x$ and $y$ in the search space $\{0,1\}^{n}$. For example, $x \preceq y$ if and only if $f(x) \preceq f(y)$.

\subsection{The Multi-objective EAs}

All the single individual multi-objective evolutionary algorithms considered in this paper are instantiations of the following scheme.

Choose $x$ uniformly from $\{0,1\}^{n}$.

\section{Repeat}

Apply mutation to $x$ to obtain $x^{\prime}$.

If selection favors $x^{\prime}$ over $x$

then $x:=x^{\prime}$.

The algorithms differ in the choice of the mutation operator and in the choice of the selection operator. Two different mutation operators are considered. The local mutation operator flips a randomly chosen bit of $x$. The global mutation operator flips each bit of $x$ independently with probability $1 / n$. In the singleobjective case, the objective function $f$ establishes a total order in the search space and selection favors $x^{\prime}$ over $x$ if $f\left(x^{\prime}\right) \geq f(x)$. Then, we obtain randomized local search (RLS) and the (1+1) EA with the local and global search operator, respectively.

For multi-objective problems, there are several options when to favor the offspring $x^{\prime}$ over the parent $x$. In this work, we consider four different selection 
operators. The weakest selection operator favors $x^{\prime}$ over $x$ if $x^{\prime}$ weakly dominates $x$, or $x^{\prime}$ and $x$ are incomparable. The weak selection operator favors $x^{\prime}$ over $x$ if $x^{\prime}$ weakly dominates $x$. The strong selection operator favors $x^{\prime}$ over $x$ if $x^{\prime}$ dominates $x$. Finally, we define the $\varepsilon$-constraint selection operator for two criteria problems as follows. (See [8] for the general definition with $m$ criteria.) If $f_{1}(x)<\varepsilon$, then the operator favors $x^{\prime}$ over $x$ if $f_{1}\left(x^{\prime}\right) \geq f_{1}(x)$. If $f_{1}(x) \geq \varepsilon$, then the operator favors $x^{\prime}$ over $x$ if $f_{1}\left(x^{\prime}\right) \geq \varepsilon$ and $f_{2}\left(x^{\prime}\right) \geq f_{2}(x)$. Informally, the idea of the $\varepsilon$-constraint selection is to turn the first objective into a constraint, such that only solutions $x$ with $f_{1}(x) \geq \varepsilon$ are feasible. So the primary goal is to minimize the constraint violation and then maximize the function $f_{2}$.

By the different choices of the mutation and the selection operators, we obtain eight different single individual MOEAs as summarized in Table 1. To obtain different Pareto optimal solutions, a single objective algorithm is run repeatedly, either sequentially (re-starts) or in parallel (multi-starts), and the hope is that not all runs will end up with the same solution. In case of the $\varepsilon$-constraint selection operator, it is necessary to vary the parameter $\varepsilon$ between runs.

\begin{tabular}{lll} 
& local & global \\
\hline weakest & $\mathrm{RLS}_{\text {weakest }}$ & $(1+1) \mathrm{EA}_{\text {weakest }}$ \\
weak & $\mathrm{RLS}_{\text {weak }}$ & $(1+1) \mathrm{EA}_{\text {weak }}$ \\
strong & $\mathrm{RLS}_{\text {strong }}$ & $(1+1) \mathrm{EA}_{\text {strong }}$ \\
$\varepsilon$-constr. & $\mathrm{RLS}_{\varepsilon \text {-constr. }}$ & $(1+1) \mathrm{EA}_{\varepsilon \text {-constr. }}$ \\
\hline
\end{tabular}

Table 1. The single individual algorithms considered according to selection and mutation operator.

We compare the single individual algorithms with the population based algorithm SEMO which was introduced in [8]. The idea of SEMO is to keep a population of incomparable individuals. In each step, an offspring is produced and added to the population if it is not dominated by some individual in the population. Afterwards it may be necessary to remove individuals from the population that are weakly dominated by the new individual.

$$
\begin{aligned}
& P:=\{x\}, \text { where } x \text { is uniformly chosen from }\{0,1\}^{n} . \\
& \text { Repeat } \\
& \text { Choose } x \text { uniformly from } P \text {. } \\
& \text { Apply mutation to } x \text { to obtain } x^{\prime} . \\
& \text { If } x^{\prime} \text { is not dominated by any individual in } P \\
& \text { then add } x^{\prime} \text { to } P \text {, and remove all individuals } \\
& \text { weakly dominated by } x^{\prime} \text { from } P \text {. }
\end{aligned}
$$

In this paper, local SEMO refers to SEMO with the local mutation operator and analogously for global SEMO. The local SEMO and the global SEMO can be considered as multi-objective counterparts to (single-objective) RLS and the $(1+1)$ EA, respectively. Both variants of SEMO have been the subject of theoretical runtime analyses $([5,8,9])$. 


\section{The Objective Function}

The idea is to devise an objective function that partitions the search space into $k$ paths, each leading to some Pareto optimal solution. The search points on a path form plateaus such that it is difficult to proceed along a path, i.e., to reach the next plateau dominating the previous plateau. But there is always a short distance to another path. The idea is that the single-objective algorithms will spend most of the time jumping between paths, instead of improving on a single path, or they will spend much time to overcome a plateau. The hope is that SEMO will quickly produce a population of $k$ individuals, one individual for each path, and these individuals will advance in parallel to their respective optima.

Let $n=k \cdot m$ and $x$ a bit string of length $n$. We say that bit string $x$ is divided into $k$ blocks, where each block has length $m \geq 2$.

Definition 1 (Block Value, Active Block). Given a search point $x \in$ $\{0,1\}^{k \cdot m}$ and an integer $i, 0 \leq i \leq k-1$. Then the $i$ th block value of $x$, denoted $|x|_{i}$, is defined as

$$
|x|_{i}:=\sum_{j=m \cdot i}^{m \cdot(i+1)-1} x_{j}
$$

The active block of a search point $x$ is the left-most block with lowest block value. The number

$$
j:=\underset{0 \leq i \leq k-1}{\operatorname{argmin}}\left\{|x|_{i}\right\}
$$

denotes the active block index. ${ }^{3}$

Table 2 gives examples of block values and active blocks, where $k=4$ and $m=3$. The following multi-objective function is essentially ONEMAX defined on the active block, weighted differently with respect to each objective as to create different Pareto optimal solutions.

Definition 2 (Objective Function). For all search points $x$ the objective function $f:\{0,1\}^{n} \rightarrow \mathbb{N} \times \mathbb{N}$ is defined by

$$
f(x):=\left(f_{1}(x), f_{2}(x)\right)
$$

where

$$
f_{1}(x):=2^{j \cdot m} \cdot\left(|x|_{j}+1\right) \quad \text { and } \quad f_{2}(x):=2^{(k-j-1) \cdot m} \cdot\left(|x|_{j}+1\right)
$$

and $j$ is the active block index of $x$. The aim is to maximize $f$.

\footnotetext{
3 The definition of the active block is equivalent to the following: $j$ is the active block index of $x$ if and only if $|x|_{j}<|x|_{i}$ for all $i, 0 \leq i<j$ and $|x|_{j} \leq|x|_{i}$ for all $i$, $j<i<k$.
} 


\begin{tabular}{|c|c|c|c|c|c|c|}
\hline$x$ & $|x|_{0}$ & $|x|_{1}$ & $|x|_{2}$ & $|x|_{3}$ & $j$ & $f(x)$ \\
\hline$\underline{000} 000000000$ & 0 & 0 & 0 & 0 & 0 & $(1,512)$ \\
\hline $111011 \underline{010} 001$ & 3 & 2 & 1 & 1 & 2 & $(128,16)$ \\
\hline$\underline{010} 111011001$ & 1 & 3 & 2 & 1 & 0 & $(2,1024)$ \\
\hline $111111111 \underline{110}$ & 3 & 3 & 3 & 2 & 3 & $(1563,3)$ \\
\hline $111 \underline{110} 111111$ & 3 & 2 & 3 & 3 & 1 & $(24,192)$ \\
\hline$\underline{111} 111111111$ & 3 & 3 & 3 & 3 & 0 & $(4,2048)$ \\
\hline
\end{tabular}

Table 2. Examples of block values, active blocks (underlined), and objective function values.

The following proposition states that search points with the same active block index are comparable whereas search points with distinct block indices are incomparable.

Proposition 1. Let $x, y \in\{0,1\}^{n}$ be two search points with active block index $i$ and $j$, respectively.

1. If and only if $i=j, x$ and $y$ are comparable.

2. Moreover, if $i=j, x \preceq y$ is equivalent to $|x|_{i} \leq|y|_{j}$.

Proof. Assume $i=j$. Then the objective function values of $x$ and $y$ depend only on $|x|_{i}$ and $|y|_{j}$, respectively. Since $f_{1}$ and $f_{2}$ are strictly increasing functions, $x$ weakly dominates $y$ or vice versa. Moreover, $|x|_{i} \leq|y|_{j}$ is equivalent to $f_{1}(x) \leq f_{1}(y) \wedge f_{2}(x) \leq f_{2}(y)$ and the latter is equivalent to $x \preceq y$.

It remains to show that if $x$ and $y$ are comparable then $i=j$. W.l.o.g. assume $x \preceq y$. Then, by $f_{1}$, the assumption implies $2^{i \cdot m}\left(|x|_{i}+1\right) \leq 2^{j \cdot m}\left(|y|_{j}+1\right)$. This is equivalent to $|x|_{i} \leq 2^{(j-i) \cdot m}\left(|y|_{j}+1\right)-1$. For $i>j$, the right-hand side of the last inequality is at most $2^{-m}(m+1)-1<0$ as $m \geq 2$. Hence, $i>j$ is impossible. For $i<j$, considering $f_{2}$ leads to the same contradiction.

We can now describe the Pareto front and the Pareto set. It follows from Proposition 2 that the Pareto front has cardinality $k$.

Proposition 2. Let $n=m \cdot k$. For all integers $i, 1 \leq i \leq k-1$, define the sets

$$
\begin{aligned}
& X_{0}^{*}:=\left\{1^{n}\right\}, \quad \text { and } \\
& X_{i}^{*}:=\left\{\left.x \in\{0,1\}^{n}|| x\right|_{i}=m-1,\right. \\
& \forall j, 0 \leq j<i, \quad|x|_{j}=m, \text { and } \\
& \left.\forall j, i<j<k, \quad|x|_{j} \geq m-1\right\} .
\end{aligned}
$$

Furthermore, for all integers $i, 1 \leq i \leq k-1$, define the points

$$
\begin{aligned}
& F_{0}^{*}:=\left(m+1,2^{(k-1) \cdot m} \cdot(m+1)\right), \quad \text { and } \\
& F_{i}^{*}:=\left(2^{i \cdot m} \cdot m, 2^{(k-i-1) \cdot m} \cdot m\right) .
\end{aligned}
$$


Then the Pareto set $X^{*}$ and the Pareto front $F^{*}$ of the bi-objective function defined in Definition 2 with parameters $m$ and $k$ are given by

$$
X^{*}=\bigcup_{i=0}^{k-1} X_{i}^{*} \quad \text { and } \quad F^{*}=\bigcup_{i=0}^{k-1}\left\{F_{i}^{*}\right\} .
$$

Furthermore, $f^{-1}\left(F_{i}^{*}\right)=X_{i}^{*}$.

Proof. We first prove that for all $i, 0 \leq i<k$, the set $X_{i}^{*}$ contains all Pareto optimal search points with active block index $i$. By Proposition 1, two search points are comparable if and only if they have the same active block index. Restricted to search points $x$ with a fixed active block index $i$, functions $f_{1}$ and $f_{2}$ are strictly increasing in the active block value $|x|_{i}$. Therefore, to prove that $X_{i}^{*}$ contains exactly the Pareto optimal search points with active block index $i$, it suffices to prove the claim that $X_{i}^{*}$ contains exactly the search points with maximal active block value and active block index $i$. The highest active block value for search points with active block index 0 is $m$, and this value is only reached by search point $1^{n}$. Hence, the claim holds for set $X_{0}^{*}$. We now prove that the claim also holds for all other sets $X_{i}^{*}, 0<i<k$. The highest active block value for search points with active block index different from 0 is $m-1$. By definition, set $X_{i}^{*}$ contains only search points with active block index $i$ and active block value $m-1$. To show that $X_{i}^{*}$ contains all such search points, assume that $y$ is any search point with active block index $i$ and active block value $m-1$. It then follows from Definition 1 that $|y|_{i}=m-1$, that for all $j, 0 \leq j<i$, $|y|_{j}>|y|_{i}=m-1$, and that for all $j, i<j<k,|y|_{j} \geq|y|_{i}=m-1$. Hence, search point $y$ must belong to set $X_{i}^{*}$. The claim therefore also holds for sets $X_{i}^{*}$, $0<i<k$.

By the discussion above, and the fact that the active block index is defined for all search points, all Pareto optimal search points must be member of one of the sets $X_{i}^{*}, 0 \leq i<k$. Hence, all Pareto optimal search points are members of the set $X^{*}$.

We now prove that the objective function $f$ maps all elements of $X^{*}$ to a point in $F^{*}$, and that for every point $y$ in $F^{*}$, there exists a search point $x$ in $X^{*}$ such that $f(x)=y$. The active block value of $1^{n}$ is $m$, so $f\left(1^{n}\right)=$ $\left(m+1,2^{(k-1) \cdot m} \cdot(m+1)\right)$, and hence the element in $X_{0}^{*}$ is mapped to point $F_{0}^{*}$. For all $i, 0<i<k$, all elements $x$ in $X_{i}^{*}$ have active block value $m-1$, and therefore $f(x)=\left(2^{i \cdot m} \cdot m, 2^{(k-i-1) \cdot m} \cdot m\right)$. Hence, for all $i, 0<i<k$, all elements in $X_{i}^{*}$ are mapped to point $F_{i}^{*}$.

We now show that the preimage of $F^{*}$ is contained in $X^{*}$. Obviously, the equality $f^{-1}\left(F_{0}^{*}\right)=X_{0}^{*}$ holds. Now assume that $i \geq 1$ and suppose that $x$ with active block index $j \neq i$ is contained in the preimage of $F_{i}^{*}$, i. e., $f(x)=F_{i}^{*}$. By considering the first objective, this implies $2^{j \cdot m}\left(|x|_{j}+1\right)=2^{i \cdot m} m$ which is equivalent to $|x|_{j}=2^{(i-j) m} m-1$. If $i>j$ the last equality implies $|x|_{j}>m$ and if $i<j$ it implies $|x|_{j}<0$. Both cases are impossible.

A popular method to solve a multi-objective problem $g=\left(g_{1}, \ldots, g_{m}\right)$ is to solve the single-objective problem $g^{\prime}:=\sum_{i} w_{i} \cdot g_{i}$ instead, where the scalar ob- 
jective function $g^{\prime}$ is a weighted sum of the original vector valued function $g$. The hope is to find different Pareto optima for different parameter settings $w_{i}>0$. However, it is well-known that such linear aggregation functions fail for the non-convex parts of the Pareto front of $g$. Pareto optimal vectors which are not located on the convex hull of the solutions in the objective space cannot be detected for any setting of the weights. For our bi-objective problem $f$ presented in Definition 2, maximizing $w_{1} f_{1}+w_{2} f_{2}$ is equivalent to maximizing $w f_{1}+(1-w) f_{2}$ where $w \in[0,1]$. As the entire Pareto front of $f$ is non-convex, any choice of $w$ allows only to detect the solutions that maximize either $f_{1}$ or $f_{2}$. Hence, all methods that require a convex Pareto front are not applicable to $f$.

\section{Weak And Strong Selection}

We show that there is a large fraction of the Pareto front such that, with an overwhelming probability, the algorithms $\mathrm{RLS}_{\text {weak }},(1+1) \mathrm{EA}_{\text {weak }}, \mathrm{RLS}_{\text {strong, }}$, and $(1+1) \mathrm{EA}_{\text {strong }}$ have to be started $e^{\Omega(n)}$ times before finding any Pareto optimal point from this fraction. The next proposition follows directly from Proposition 1.

Proposition 3. All search points selected by either the weak selection operator, or the strong selection operator have the same active block index as the initial search point.

Theorem 1. Let $n=m \cdot k$, where $m$ is a constant, and $A$ is any of the algorithms $R L S_{\text {weak }},(1+1) E A_{\text {weak }}, R L S_{\text {strong }}$, and (1+1) EA $A_{\text {strong. }}$. Then, for all constants $\alpha, 0<\alpha<1$, there is a subset $F_{\alpha}^{*}$ of the Pareto front $F^{*}$ with cardinality $\left|F_{\alpha}^{*}\right| \geq \alpha\left|F^{*}\right|-1$ such that the probability that algorithm A needs less than $e^{c \cdot n}$ runs to find any Pareto optimal point in $F_{\alpha}^{*}$ is bounded by $e^{-\Omega(n)}, c>0$ a sufficiently small constant.

Proof. Define $F_{\alpha}^{*}:=\cup_{i \geq(1-\alpha) k}^{k-1}\left\{F_{i}^{*}\right\}$, where $F_{i}^{*}$ is as in Proposition 2. The Pareto front contains $k$ points, and $F_{\alpha}^{*}$ contains $\alpha k$ elements, so the cardinality of $F_{\alpha}^{*}$ is greater than $\alpha\left|F^{*}\right|-1$. All search points in the pre-image of $F_{\alpha}^{*}$ have active block indices at least $(1-\alpha) k$. By Proposition 3, in order to find a search point with an active block index that high, the initial search point must have active block index at least $(1-\alpha) k$. To get such a high active block index, it is necessary that all of the first $\lfloor(1-\alpha) k\rfloor$ blocks have block value unequal to 0 . An upper bound on the probability that the active block index is higher than $(1-\alpha) k$ is therefore $\left(1-(1 / 2)^{m}\right)^{(1-\alpha) \cdot k}=e^{-\Omega(n)}$ as $m$ is a constant and, therefore, $k=\Theta(n)$. Furthermore, the probability that the event occurs within $e^{c \cdot n}$ runs is no more than $e^{c \cdot n} \cdot e^{-\Omega(n)}=e^{-\Omega(n)}$, for $c>0$ a sufficiently small constant.

Both weak and strong selection turn out to be inadequate. The active block index of the initial search point will almost always be low, but these selection operators do not allow changing the active block index in a run. The weakest selection operator alleviates this problem by allowing to change the active block index; however, we show in the next section that this is not sufficient. 


\section{$5 \quad$ Weakest Selection}

We prove that $\mathrm{RLS}_{\text {weakest }}$ and the $(1+1) \mathrm{EA}_{\text {weakest }}$ need with overwhelming probability an exponential time to find any Pareto optimal solution. The idea is the following. All Pareto optimal solutions have at most $k 0$-bits. We show that if the number of 0 -bits is close to $k$, it is unlikely to lose another 0-bit in the next accepted step and it is much more likely to gain new 0-bits. Thus, there is a strong tendency to increase the number of 0-bits. To show that the probability of increasing the number 0-bits is high, we first show that there are many 1-bits that can be turned into zeroes.

Proposition 4. Let $x$ be any search point with $\ell \geq m$ 1-bits. There are $\ell-m$ 1-bits in $x$ such that flipping any of these 1-bits produces a search point $x^{\prime}$ which is not dominated by $x$.

Proof. There are at most $m$ 1-bits in the active block of $x$. Assume that any of the remaining $\ell-m$ 1-bits flip. If the active block index changes, $x$ and $x^{\prime}$ are incomparable (Proposition 1). Otherwise, the active block value remains unchanged implying that $x^{\prime}$ cannot be dominated by $x$.

\section{$5.1 \quad$ RLS}

The analysis of how the number of 0-bits evolves over time will be based on a simple Markov process also known as the gambler's ruin problem. A gambler owns an initial capital of $a$ dollars and plays against an adversary whose initial capital is $b$ dollars. The gambler wins and loses a round with probability $p$ and $1-p$, respectively. If he loses a round, he pays a dollar to the adversary and otherwise receives a dollar from the adversary. They continue the game until one player is ruined and the winner is the player who then owns the combined capital $a+b$. For a proof of the next theorem see, e.g., [4] or [1].

Theorem 2. For $p \neq 1 / 2$, the probability that the gambler wins is

$$
\frac{1-t^{a}}{1-t^{a+b}}
$$

where $t:=(1-p) / p$. Consequently, for $p>1 / 2$, this probability is at least $1-t^{a}$.

Theorem 3. For $k \geq 4$ and $m \geq 5$, the expected time for $R L S_{\text {weakest }}$ to find any Pareto optimal solution is $e^{\Omega(n)}$. Moreover, there are positive constants $c$ and $c^{\prime}$ such that the probability that $R L S_{\text {weakest }}$ finds any Pareto optimal solution in $e^{c^{\prime} \cdot n}$ runs, each of $e^{c \cdot n}$ steps, is $e^{-\Omega(n)}$.

Proof. By Chernoff bounds, the initial search point has less than $n / 4$ 0-bits with an exponentially small probability of $e^{-\Omega(n)}$. We only consider the case where the first search point has at least $n / 40$-bits and wait for the first point in time where the number of 0 -bits is at most $n / 4$. In the following, we consider only situations with at most $n / 40$-bits. Then the number of 1 -bits is at least 
$3 n / 4>m$ and we can apply Proposition 4. Each mutation step of RLS either increases or decreases the number of 0-bits by 1 , but not all steps are accepted. The probability that the next step is accepted and the number of 0-bits increases is at least $(3 n / 4-m) / n=3 / 4-1 / k \geq 2 / 4$. The probability that the next step is accepted and the number of 0 -bits decreases is at most $(n / 4) / n \leq 1 / 4$. Hence, accepted steps increase and decrease the number of 0-bits with a probability of at least $p:=2 / 3$ and at most $1-p=1 / 3$, respectively. We consider the number of 0 -bits as the capital of the gambler in Theorem 2. Initially, it is $\lfloor n / 4\rfloor$ and the capital of his opponent is 1 . Then the probability that the number of 0-bits increases to $\lfloor n / 4\rfloor+1$ before it decreases to 0 is at least $1-t^{\lfloor n / 4\rfloor+1}$ where $t:=1 / 2$. We are interested in the probability to produce a Pareto optimal point. A Pareto optimal point has less than $k 0$-bits. Hence, we consider the gambler ruined as soon as his capital reaches $k$ dollars. This is equivalent to reducing his initial capital by $k$. Since $m \geq 5$, we have $k \leq n / 5$ and obtain an upper bound of $t^{\lfloor n / 4\rfloor-k+1}=e^{-\Omega(n)}$ for the probability to reach a Pareto optimal point before a point with at least $n / 40$-bits again. Hence, we can apply this argument repeatedly such that for a sufficiently small constant $c, e^{c \cdot n}$ repetitions of the game are successful with a probability of only $e^{-\Omega(n)}$. Taking into account the probability that the initial step is not as desired leads to the result that a run of $e^{c \cdot n}$ steps is successful with a probability of only $e^{-\Omega(n)}$ and leads to the claimed expected runtime.

We now consider independent runs of $\mathrm{RLS}_{\text {weakest }}$, i.e., sequential runs (restarts) or parallel runs (multi-starts). If each of $e^{c^{\prime} \cdot n}$ runs includes up to $e^{c \cdot n}$ steps, the probability that any of these runs is successful is at most $e^{c^{\prime} \cdot n} \cdot e^{-\Omega(n)}=$ $e^{-\Omega(n)}$ if $c^{\prime}>0$ is sufficiently small. Hence, independent runs of $\operatorname{RLS}_{\text {weakest }}$ do not help to increase the success probability substantially.

\section{$5.2(1+1)$ EA}

The global mutation operator of the $(1+1) \mathrm{EA}_{\text {weakest }}$ may flip many bits in one step and increase or decrease the number of 0-bits by large values. Although the probability of a large change in a single step is rather low, such a step is not unlikely to happen in a run including exponentially many steps. Therefore, we have to take large changes into account. The following drift theorem provides a general technique for proving exponential lower bounds on the first hittingtime in Markov processes. It serves as a counterpart to Theorem 2. We apply a result due to [7] that goes back to [6]. Analyzing the proof in [7], it follows immediately that it includes a stronger result than stated, namely a result on the success probability to reach a state with certain properties and not only the expected waiting time. We state this result in Theorem 4.

Theorem 4 (Drift Theorem). Let $X_{0}, X_{1}, X_{2}, \ldots$ be the random variables describing a Markov process over the state space $S$ and $g: S \rightarrow \mathbb{R}_{0}^{+}$a function that assigns to each state a non-negative real number. Pick two real numbers $a(n)$ and $b(n)$ which depend on a parameter $n$ such that $0 \leq a(n)<b(n)$ holds. 
Let the random variable $T$ denote the earliest point in time $t \geq 0$ that satisfies $g\left(X_{t}\right) \leq a(n)$.

If there are constants $\lambda>0$ and $D \geq 1$ and a polynomial $p(n)>0$ such that the four conditions

$$
\begin{aligned}
& g\left(X_{0}\right) \geq b(n) \\
& b(n)-a(n)=\Omega(n), \\
& \forall t \geq 0: E\left(e^{-\lambda\left(g\left(X_{t+1}\right)-g\left(X_{t}\right)\right)} \mid X_{t}, a(n)<g\left(X_{t}\right)<b(n)\right) \leq 1-1 / p(n) \\
& \forall t \geq 0: E\left(e^{-\lambda\left(g\left(X_{t+1}\right)-b(n)\right)} \mid X_{t}, \quad b(n) \leq g\left(X_{t}\right)\right) \leq D
\end{aligned}
$$

hold then for all time bounds $B \geq 0$

$$
\operatorname{Prob}(T \leq B) \leq e^{\lambda(a(n)-b(n))} \cdot B \cdot D \cdot p(n) .
$$

Since $\lambda(a(n)-b(n))=-\Omega(n)$ and $p(n)$ is a polynomial, the last bound is exponentially small for $B:=e^{c \cdot n}$ if $c>0$ is sufficiently small.

Theorem 5. For $4 \leq k \leq((1 / 10)-\varepsilon) n, \varepsilon<1 / 10$ a positive constant, the expected time for the $(1+1)$ EA weakest to find any Pareto optimal solution is $e^{\Omega(n)}$. Moreover, there are positive constants $c$ and $c^{\prime}$ such that the probability that the $(1+1)$ EA $A_{\text {weakest }}$ finds any Pareto optimal solution in $e^{c^{\prime} \cdot n}$ runs, each of $e^{c \cdot n}$ steps, is $e^{-\Omega(n)}$.

Proof. Let the random variable $X_{t} \in\{0,1\}^{n}$ denote the search point of the $(1+1) \mathrm{EA}_{\text {weakest }}$ at time $t \geq 0$ when applied to $f$. To apply the above drift theorem, $g\left(X_{t}\right)$ equals the number of 0 -bits of $X_{t}$. We choose $b(n):=n / 10$ and $a(n):=k=n / m$. By Chernoff bounds, the initial search point $X_{0}$ has more than $b(n)$ 0-bits with an overwhelming probability of $1-e^{-\Omega(n)}$. Thus, we only consider the case where $b(n)<g\left(X_{0}\right)$ such that the first condition is satisfied. As $k \leq((1 / 10)-\varepsilon) n$, also the second condition is met.

To check the third condition we have to bound

$$
E\left(e^{-\lambda\left(g\left(X_{t+1}\right)-g\left(X_{t}\right)\right)} \mid X_{t}, k<g\left(X_{t}\right)<n / 10\right)
$$

from above. Let $p_{j}\left(X_{t}\right)$ denote the probability that the $g$-value increases by $j$ in the next step when the current search point is $X_{t}$ and $k<g\left(X_{t}\right)<n / 10$. Then the above expectation is bounded from above by

$$
\sum_{j:=-g\left(X_{t}\right)}^{n-g\left(X_{t}\right)} e^{-\lambda \cdot j} \cdot p_{j}\left(X_{t}\right)
$$

For $j>0$, we only increase the value of the sum $(*)$ if we replace $p_{j}\left(X_{t}\right)$ with some lower bound $p_{j}$ independent of $X_{t}$ and increase $p_{0}\left(X_{t}\right)$ by $\Delta_{j}:=p_{j}\left(X_{t}\right)-p_{j}$. For all $j \geq 2$, we choose the trivial lower bounds $p_{j}:=0$. The probability $p_{1}\left(X_{t}\right)$ is lower bounded by the probability of the event that exactly one 1-bit of at least $n-b(n)-m \geq 9 n / 10-m 1$-bits flip (Proposition 4). Hence, for $k \geq 4$,

$$
\frac{9 n / 10-m}{n}(1-1 / n)^{n-1} \geq \frac{9}{10 e}-\frac{1}{k e} \geq \frac{2}{10}
$$


and we can choose $p_{1}:=2 / 10$.

For $j<0$, the value of the sum $(*)$ only increases if we replace $p_{j}\left(X_{t}\right)$ by some upper bound $p_{j}$ and decrease $p_{0}\left(X_{t}\right)$ by $\Delta_{j}:=p_{j}-p_{j}\left(X_{t}\right)$. The probability of decreasing the $g$-value by $j$ in the next step is upper bounded by the probability of the event that at least $j$ 0-bits are turned into 1-bits. Hence,

$$
\left(\begin{array}{c}
g\left(X_{t}\right) \\
j
\end{array}\right) \cdot \frac{1}{n^{j}} \leq \frac{(n / 10)^{j}}{j !} \cdot \frac{1}{n^{j}}=\frac{1}{10^{j} j !}
$$

and $p_{j}:=1 /\left(10^{j} j !\right)$ is a correct upper bound. We now consider the Markov process where all probabilities $p_{j}\left(X_{t}\right)$ are replaced with our corresponding bounds. If we pessimistically assume that the $g$-value, the number of 0-bits can decrease by any $j>0$ (i.e., also for $j>g\left(X_{t}\right)$ ) we only overestimate the probability to decrease the $g$-value. We obtain a process where the transition probabilities are independent of the $g(X)$-value. The new process with

$$
\begin{aligned}
p_{j} & :=0, \quad \text { for all } j \geq 2, \\
p_{1} & :=\frac{2}{10}, \\
p_{0} & :=1-\sum_{j \neq 0} p_{j}, \\
p_{-1} & :=\frac{1}{10}, \\
p_{-j} & :=\frac{1}{10^{j} j !}, \quad \text { for all } j \geq 2,
\end{aligned}
$$

reaches a $g$-value of at most $a(n)$ only "faster" than the original process describing the $(1+1) \mathrm{EA}_{\text {weakest }}$ applied to $f$.

It now suffices to bound the sum

$$
\begin{aligned}
& e^{-1 \cdot \lambda} p_{1}+e^{-0 \cdot \lambda} p_{0}+e^{1 \cdot \lambda} p_{-1}+\sum_{j \geq 2} e^{j \cdot \lambda} p_{-j} \\
= & \left(1-p_{1}-p_{-1}-\sum_{j \geq 2} p_{-j}\right)+e^{-\lambda} p_{1}+e^{\lambda} p_{-1}+\sum_{j \geq 2} e^{j \cdot \lambda} p_{-j} \\
= & 1-\left(\left(1-e^{-\lambda}\right) p_{1}+\left(1-e^{\lambda}\right) p_{-1}\right)+\sum_{j \geq 2}\left(e^{j \cdot \lambda}-1\right) p_{-j}
\end{aligned}
$$

for an appropriate choice of $\lambda>0$. We choose $\lambda:=(1 / 2) \ln 2$ and show that the sum $(* *)$ is at most $1-\alpha+\beta$ for positive constants $\alpha$ and $\beta$, where $\alpha>\beta$. For our choice of $\lambda$, we obtain

$$
\left(1-e^{-\lambda}\right) p_{1}+\left(1-e^{\lambda}\right) p_{-1}=: \alpha>\frac{17}{1000}
$$


and

$$
\begin{aligned}
\sum_{j \geq 2}\left(e^{j \cdot \lambda}-1\right) p_{-j} \leq \sum_{j \geq 2} \frac{2^{j / 2}}{10^{j} j !} & =\sum_{j \geq 2} \frac{(\sqrt{2} / 10)^{j}}{j !} \\
& =\exp (\sqrt{2} / 10)-1-\sqrt{2} / 10=: \beta<\frac{11}{1000} .
\end{aligned}
$$

Hence, the sum $(* *)$ is at most $1-\delta$ for a positive constant $\delta$ and we can choose a constant polynomial $p(n):=1 / \delta$.

It remains to check the last condition of Theorem 4 . We bound

$$
\begin{aligned}
& E\left(e^{\left.-\lambda\left(g\left(X_{t+1}\right)-n / 10\right)\right)} \mid X_{t}, n / 10 \leq g\left(X_{t}\right)\right) \\
\leq & E\left(e^{-\lambda\left(g\left(X_{t+1}\right)-g\left(X_{t}\right)\right)} \mid X_{t}, n / 10 \leq g\left(X_{t}\right)\right)
\end{aligned}
$$

from above and proceed analogously to the case of the third condition. We bound $p_{j}\left(X_{t}\right)$ by the trivial lower bound $p_{j}:=0$, for all positive $j$. For $j$ negative, the probability $p_{j}\left(X_{t}\right)$ is upper bounded by the probability of the event that at least $j$ 1-bits flip. The corresponding probability is at most

$$
\left(\begin{array}{c}
n \\
j
\end{array}\right) \cdot \frac{1}{n^{j}} \leq \frac{1}{j !} \leq\left(\frac{e}{j}\right)^{j} .
$$

We consider the process where

$$
\begin{aligned}
p_{j} & :=0, \quad \text { for all } j \geq 1, \\
p_{0} & :=1-\sum_{j \geq 1} p_{j}, \\
p_{-j} & :=\left(\frac{e}{j}\right)^{j}, \quad \text { for all } j \geq 1 .
\end{aligned}
$$

Now it suffices to estimate the sum

$$
\begin{aligned}
e^{-0 \cdot \lambda} p_{0}+\sum_{j \geq 1} e^{j \cdot \lambda} p_{-j} \leq & 1+\sum_{j \geq 1} 2^{j / 2}\left(\frac{e}{j}\right)^{j} \\
\leq & 1+\sum_{j \geq 1}\left(\frac{\sqrt{2} e}{j}\right)^{j}=O(1)+\sum_{j \geq 12}\left(\frac{1}{2}\right)^{j}=O(1) .
\end{aligned}
$$

Hence the last sum is bounded by some positive constant $D$.

By Theorem 4, the probability that a state with less than $k$ 0-bits is reached in $B:=e^{c \cdot n}$ steps is $e^{-\Omega(n)}$ if $c$ is sufficiently small. Taking into account the probability that the initial search point has at least $n / 10$ 0-bits leads to the success probability of $e^{-\Omega(n)}$ in a run of up to $e^{c \cdot n}$ steps. This result implies the claimed expected runtime and, by the same arguments as presented at the end of the proof of Theorem 3, a success probability of $e^{-\Omega(n)}$ for $e^{c^{\prime} \cdot n}$ independent runs. 


\section{$6 \varepsilon$-constraint Selection}

Proposition 5. Let $n=m \cdot k$ and let $A$ be any of the algorithms $R L S_{\varepsilon \text {-constr. }}$ and $(1+1) E A_{\varepsilon \text {-constr. }}$. For a constant $\beta, 0<\beta<1$, define the stochastic process $X_{1}, \ldots, X_{s}$, where $X_{i} \in\{0,1\}^{(1-\beta) m k}$ is the $(1-\beta) m k$-bit long suffix of the search point in step $i$ of algorithm $A$ working on the objective function defined in Definition 2. Then, given that the search points in the first $s$ steps of $A$ have active block indices less than $\beta k$ and active block values 0 , each of the vectors $X_{i}$ is uniformly distributed over $\{0,1\}^{(1-\beta) m k}$.

Proof. The proof is by induction over the number of iterations $i$. The initial search point is sampled uniformly at random, so the statement trivially holds for the base case $i=1$. Assume that the vectors $X_{1}, \ldots, X_{i}$ are uniformly distributed over $\{0,1\}^{(1-\beta) m k}$. Because the active block index of $X_{i}$ is less than $\beta k$ and the active block value is 0 , acceptance of a new search point does not depend on the $(1-\beta) m k$-bit long suffix of the search point. The global and the local mutation operator applied to a uniformly distributed suffix, produces a uniformly distributed suffix. (See e.g., [3]).

Theorem 6. Let $n=m \cdot k$, where $m \geq 2$ is a constant and $\alpha, 0<\alpha<1$, is an arbitrary constant. Then there exists constants $c, c^{\prime}>0$, and a subset $F_{\alpha}^{*}$ of the Pareto front $F^{*}$ with cardinality $\left|F_{\alpha}^{*}\right| \geq \alpha \cdot\left|F^{*}\right|$ such that the probability that $e^{c^{\prime} \cdot n}$ runs, each of $e^{c \cdot n}$ steps, of $R L S_{\varepsilon \text {-constr. or }}(1+1) E A_{\varepsilon \text {-constr. find any }}$ Pareto optimal point of $F_{\alpha}^{*}$ is $e^{-\Omega(n)}$. The parameter setting $\varepsilon$ is allowed to change between runs.

Proof. Define $F_{\alpha}^{*}:=\cup_{i=0}^{\alpha \cdot k-1}\left\{F_{i}^{*}\right\}$, where $F_{i}^{*}$ is as in Proposition 2. We call a run bad when the initial search point has active block index higher than $\alpha k$, or has active block value higher than 0 . The probability of the first case is upper bounded by the probability of the event that all the first $\alpha k$ blocks have block values different from 0 , and the second case is upper bounded by the probability of the event that all blocks have block values different from 0 . Because the second event implies the first event, the probability of a bad run is no more than $\left(1-(1 / 2)^{m}\right)^{\alpha k}=e^{-\Omega(n)}$.

Assume now that the initial search point has active block index less than $\alpha k$ and active block value 0 . No Pareto optimal search point has active block value 0 when $m \geq 2$. We lower bound the optimization time by analyzing the time until the search point for the first time has active block value at least 1 . We say that the algorithm is in the constraint-minimization state when the search point $x$ has function value $f_{1}(x)<\varepsilon$, and in the maximization state when the search point $x$ has function value $f_{1}(x) \geq \varepsilon$. In the maximization state, a search point $x$ will be replaced by a search point $x^{\prime}$ if and only if $f_{2}\left(x^{\prime}\right) \geq f_{2}(x)$ and $f_{1}\left(x^{\prime}\right) \geq \varepsilon$. Consequently, the algorithm will never leave the maximization state once entered, and the active block index can only decrease in this state. (See Definition 2.) The maximal active block index during a run will, therefore, never be higher than the active block index in the first step after the algorithm has entered the maximization state. We will show that with overwhelming probability, the highest active block index will never be higher than $(k / 2)(1+\alpha)$. 
We divide the search point into three parts as shown in Figure 1. We first divide the string into an $\alpha k$ blocks long prefix and a $(1-\alpha) k$ blocks long suffix. Then, we divide the suffix into two almost equally long parts, each approximately $(k / 2)(1-\alpha)$ blocks long. The last part now begins at block index $\alpha k+(k-\alpha k) / 2=(k / 2)(1+\alpha)$.

Assume first that $\varepsilon<2^{\alpha m k}$. As long as the algorithm is in the constraint minimization state, the active block index is less than $\alpha k$. (See Definition 2.) Therefore, by Proposition 5, the $(1-\alpha) k$ blocks long suffix will be uniformly distributed. Furthermore, in the step when the algorithm enters the maximization state, the blocks in the interval from $\alpha k$ to $(k / 2)(1+\alpha)$ will also be uniformly distributed. Hence, the probability that the first search point in the maximization state has active block index higher than $(k / 2)(1+\alpha)$ is upper bounded by the probability that all the blocks in the interval from $\alpha k$ to $(k / 2)(1+\alpha)$ have block values different from 0 . By the uniform distribution, the probability of this event is no more than $\left(1-(1 / 2)^{m}\right)^{(k / 2)(1-\alpha)}=e^{-\Omega(n)}$. Therefore, with overwhelming probability, the active block index during the entire run will be no more than $(k / 2)(1+\alpha)$.

We now consider the second case where $\varepsilon \geq 2^{\alpha k m}$. In this case, all elements in $F_{\alpha}^{*}$ violate the $\varepsilon$-constraint. If the active block index becomes higher than $\alpha k$, none of the search points in $F_{\alpha}^{*}$ can be found. We optimistically assume that the active block index during the entire run will never be higher than $\alpha k$ when $\varepsilon \geq 2^{\alpha k m}$.

Hence, for both cases, we can now assume that the active block index is less than $(k / 2)(1+\alpha)$ during the entire run. So by Proposition 5 , the suffix $X_{i}$ corresponding to the last $(k / 2)(1-\alpha)-1$ blocks of the search point will be uniformly distributed over the set $\{0,1\}^{m((k / 2)(1-\alpha)-1)}$. We say that the vector $X_{i}$ is good if all blocks in $X_{i}$ have block values different from 0 . Because $X_{i}$ is uniformly distributed, $\operatorname{Prob}\left(" X_{i}\right.$ is good") $=\left(1-(1 / 2)^{m}\right)^{(k / 2)(1-\alpha)-1}=e^{-\Omega(n)}$. To reach active block value 1 within $s$ steps, at least one of the variables $X_{1}, \ldots, X_{s}$ must be good. By union bound, the probability of at least one good variable $X_{i}$ during a run of length $s:=e^{c n}$ is no more than $\operatorname{Prob}\left(\cup_{i=1}^{e^{c n}}\right.$ " $X_{i}$ is $\left.\operatorname{good} "\right) \leq e^{c n} \cdot e^{-\Omega(n)}=$ $e^{-\Omega(n)}$ for a sufficiently small constant $c$.

Furthermore, $e^{c^{\prime} \cdot n}$ runs, each of length $e^{c \cdot n}$, will be successful with probability $e^{-\Omega(n)}$ for a sufficiently small constant $c^{\prime}>0$.

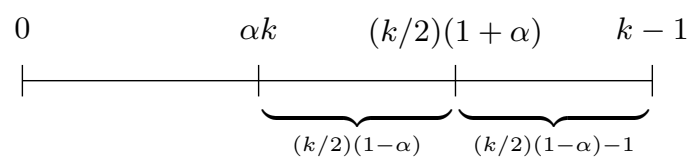

Figure 1. Active block index.

\section{SEMO}

We prove that within polynomial time, the SEMO population covers the entire Pareto front on the problem defined in Definition 2. The idea is the following. The 
problem consists of $k$ independent paths, one path for each block, with Pareto optimal solutions at the end of each path. However, to progress to a higher level on a path, a large plateau must be overcome. We show that the individuals in the SEMO population will be distributed over these paths, with at most one individual per path. SEMO will, thereby, optimize the paths in parallel, such that no gain along any path is lost. The individuals in the population of SEMO are pairwise incomparable. Hence, the following proposition is a consequence of Proposition 1.

Proposition 6. For each block index $j, 0 \leq j \leq k-1$, a population in SEMO has at most one element with active block index $j$.

We introduce a concept called the active path of the population to analyze the parallel improvements along each path. Informally, the active path number corresponds to the active block index on which SEMO has advanced the most, and the active path value designates how far on this path SEMO has advanced.

Definition 3 (Active Path). Let $x_{1}, x_{2}, \ldots, x_{r}$ be the individuals in a SEMO population, and $j_{1}, j_{2}, \ldots, j_{r}$ their respective active block indices. Then the active path value $v$ of a population is the maximal active block value in the population, i.e.,

$$
v:=\max _{1 \leq i \leq r}\left\{\left|x_{i}\right|_{j_{i}}\right\},
$$

and the active path number $t$ of the population is the highest active block index among the individuals having active block value $v$, i.e.,

$$
t:=\max _{1 \leq i \leq r}\left\{\left.j_{i}|| x_{i}\right|_{j_{i}}=v\right\}
$$

(Note that, by Proposition 6, there is only one individual in the population with active block index $t$.)

Table 3 gives five examples of active path number and active path value of a population. Each row describes a population, and each population has three individuals. The last two columns in the table give the active path number $t$ and the active path value $v$ of the corresponding population. Additionally, the active path representative in each population is framed.

When the active path value of a population is $m$, the population must contain the Pareto optimal solution $1^{n}$. After the Pareto optimal solution $1^{n}$ has been found, SEMO will quickly discover the rest of the Pareto front. Our approach to analyze SEMO, therefore, focuses on the time it takes to increase the active path value to $m$.

Proposition 7. The active path value never decreases. If the active path number decreases then the active path value increases.

Proof. Suppose that the active path value decreases, and the old active path was represented by individual $x$. Then individual $x$ cannot be member of the new population. Hence, the new population must contain a new element $x^{\prime}$ such that 


\begin{tabular}{|c|c|c|c|c|c|}
\hline$P$ & Individual 0 & Individual 1 & Individual 2 & $t$ & $v$ \\
\hline 1 & $\underline{00} 0000$ & $01 \underline{00} 00$ & 010100 & 2 & 0 \\
\hline 2 & $\begin{array}{|lll|}01 & 01 & 01\end{array}$ & $01 \underline{00} 00$ & $0101 \underline{00}$ & 0 & 1 \\
\hline 3 & $\underline{01} 0101$ & $11 \underline{01} 01$ & $0101 \underline{00}$ & 1 & 1 \\
\hline 4 & $\underline{01} 0101$ & $11 \underline{01} 01$ & $\begin{array}{lll}11 & 11 & 01 \\
\end{array}$ & 2 & 1 \\
\hline 5 & $\underline{11} 11111$ & $11 \underline{01} 01$ & $1111 \underline{01}$ & 0 & 2 \\
\hline
\end{tabular}

Table 3. Examples of the active path concept.

$x \preceq x^{\prime}$. Proposition 1 implies that $|x|_{j} \leq\left|x^{\prime}\right|_{j}$, which contradicts that the active path value decreases.

For the second claim, let the old active path number $i$ be represented by the search point $x$. If the active path number decreases then the new active path must be represented by the search point $x^{\prime}$, having active block index $j, 0 \leq j<i$. By Proposition 1 , the search point $x$ will remain in the new population. The only way the new search point $x^{\prime}$ can be the new active path representative is when $x^{\prime}$ has higher active block value than $x$. This means that the active path value must increase.

Proposition 8. The expected time to increase the active path value is bounded above by the expected time to change the active path number $k$ times.

Proof. Since there are $k$ different blocks, the maximal number of times the active path number can increase without being decreased is $k-1$. Hence, by Proposition 7 , after $k$ active path number changes, the active path value must have increased at least once.

Theorem 7. The expected time until the SEMO population covers the Pareto front is $O\left(n k^{2} \log m\right)$.

Proof. Our analysis will be based on 1-bit-mutations only. Since the probability that a specified bit flips is at least $1 / n$ and $1 /(e n)$ for the local and the global mutation operator, respectively, the waiting time for a specific 1-bit-mutation is only larger for the global SEMO. Consequently, it suffices to derive upper bounds on the runtime of the global SEMO.

We divide the optimization process into two consecutive phases. The first phase begins when the algorithm starts and ends when the population for the first time contains the Pareto optimal solution $1^{n}$. Thereafter, the second phase starts and it lasts until the entire Pareto front is covered.

In the first phase, the active path value must be increased at most $m$ times because an active path value of $m$ implies that the population includes the individual $1^{n}$. By Proposition 8 , at most $k$ active path number changes suffice to increase the active path value once. We call a step successful if

1. the active path number increases, or

2. the active path value increases. 
We claim that a step is successful if it first chooses the individual $x$ representing the active path (i.e., its active block index equals the active path number) and then flips one or more of the 0 -bits in the active block to obtain $x^{\prime}$. Two cases must be considered, either $x$ and $x^{\prime}$ have the same active block index, or they do not.

In the case that $x$ and $x^{\prime}$ have the same active block index, then $x^{\prime}$ clearly dominates $x$ because $x^{\prime}$ has more 1-bits in its active block. Hence, $x^{\prime}$ replaces $x$ in the new population and the active path value increases.

Now, assume that $x$ and $x^{\prime}$ have different active block indices $i$ and $j$, respectively. We first show that $x^{\prime}$ will be accepted. If the new search point $x^{\prime}$ is not accepted, then there must exist a search point $y$ in the population which strictly dominates $x^{\prime}$. Proposition 1 implies that $y$ has active block index $j$, and that $\left|x^{\prime}\right|_{j}<|y|_{j}$. Furthermore, because $i$ is the active block index in $x$, and $x^{\prime}$ by assumption differs from $x$ in block $i$ only, we have $|x|_{i} \leq|x|_{j}=\left|x^{\prime}\right|_{j}$. However, the last inequality implies that $|x|_{i}<|y|_{j}$, which contradicts that $x$ was the active path representative in the old population. The search point $x^{\prime}$ will, therefore, be accepted.

If $i>j$ then $|x|_{i}<|x|_{j}=\left|x^{\prime}\right|_{j}$ because $i$ is the active block index of $x$, and $x$ and $x^{\prime}$ do not differ in block $j$. The search point $x^{\prime}$ will be the new active path representative and the active path value increases. Analogously, $i<j$ implies $|x|_{i} \leq|x|_{j}=\left|x^{\prime}\right|_{j}$. Hence, $x^{\prime}$ has at least as high active block value as $x$, and $x^{\prime}$ has higher active block index than $x$. The search point $x^{\prime}$ will be the new active path representative and the active path number will increase.

Now we estimate the probability of a successful step. By Proposition 6, the probability of choosing the individual representing the active path is at least $1 / k$. Given that the active block value is $v$, the probability of flipping at least one of the $m-v 0$-bits in the active block of $x$ (and no other bits) is at least

$$
\frac{m-v}{n}\left(1-\frac{1}{n}\right)^{n-(m-v)} \geq \frac{m-v}{n}\left(1-\frac{1}{n}\right)^{n-1} \geq \frac{(m-v)}{e n}
$$

The probability of a successful step, therefore, is at least $(m-v) /(e k n)$. Using Proposition 8, the expected duration of the first phase is bounded from above by

$$
\sum_{v=0}^{m-1} k \cdot \frac{e k n}{(m-v)}=e k^{2} n \sum_{v=1}^{m} \frac{1}{v}=O\left(k^{2} n \log m\right)
$$

In the second phase, the population contains the Pareto optimal solution $1^{n}$, and it will never be removed from the population. Given that there are $i$ remaining points in the Pareto front to be discovered, the probability of selecting $x=1^{n}$ and mutating solely one 1-bit in one of the corresponding $i$ blocks is at least

$$
\frac{1}{k} \cdot \frac{i m}{n} \cdot\left(1-\frac{1}{n}\right)^{n-1} \geq \frac{i m}{e k n}=\frac{i}{e k^{2}}
$$


The expected time to find the at most $k-1$ remaining points in the Pareto front is, therefore, no more than

$$
\sum_{i=1}^{k-1} \frac{e k^{2}}{i} \leq e k^{2} \sum_{i=1}^{k} \frac{1}{i}=O\left(k^{2} \log k\right) .
$$

Hence, the expected time until SEMO covers the entire Pareto front is bounded by $O\left(k^{2} n \log m\right)$.

\section{Conclusion}

This paper introduces a simple bi-objective function to contrast two types of multi-objective EAs: population-based and single individual-based algorithms. The problem features a large number of incomparable search points and large plateaus. The runtime of the population-based algorithm SEMO is compared with the runtime of nine single individual-based approaches (eight variants from Table 1 plus the linear aggregation approach in Section 3). Table 4 summarizes the results. Among the algorithms studied, only SEMO finds the Pareto front in expected polynomial time. All single individual algorithms fail on this problem because they either too easily accept incomparable search points, or because they cannot overcome the large plateaus in the search space. SEMO is efficient on the problem because the individuals in the population collectively lead to better solutions, i. e., each individual follows a path leading to one Pareto optimal solution. The result demonstrates the importance of populations for certain types of multi-objective problems.

Our result improves an earlier result in [8] where it is shown that some simple population-based MOEAs slightly outperform the $\varepsilon$-constrained method. However, the result yields only a small polynomial runtime gap. Here, we provide an exponential gap, proving that even multi-start variants of a number of single individual-based approaches fail with overwhelming probability. In contrast, SEMO discovers all Pareto optimal solutions efficiently.

\section{References}

1. K. L. Chung. Elementary Probability Theory with Stochastic Processes. Springer, 1974 .

2. K. Deb. Multi-Objective Optimization Using Evolutionary Algorithms. Wiley, 2001.

3. S. Droste, T. Jansen, and I. Wegener. On the analysis of the $(1+1)$ evolutionary algorithm. Theoretical Computer Science, 276:51-81, 2002.

4. W. Feller. An Introduction to Probability Theory and Its Applications, volume 1. Wiley, 1968.

5. O. Giel. Expected runtimes of a simple multi-objective evolutionary algorithm. In Proc. of the 2003 Congress on Evolutionary Computation (CEC '03), volume 3, pages 1918-1925. IEEE, 2003.

6. B. Hajek. Hitting-time and occupation-time bounds implied by drift analysis with applications. Advances in Applied Probability, 14:502-525, 1982. 


\begin{tabular}{lll} 
Algorithm/Method & Instance & Expected Runtime \\
\hline weighted sums & all & $\infty$ (not applicable) \\
$\mathrm{RLS}_{\text {weakest }}$ & $k \geq 4, m \geq 5$ & $e^{\Omega(n)}$ \\
$\mathrm{RLS}_{\text {weak }}$ & $m$ constant & $e^{\Omega(n)}$ \\
$\mathrm{RLS}_{\text {strong }}$ & $m$ constant & $e^{\Omega(n)}$ \\
$\mathrm{RLS}_{\varepsilon \text {-constr. }}$ & $m \geq 2$ and constant & $e^{\Omega(n)}$ \\
$(1+1) \mathrm{EA}_{\text {weakest }}$ & $4 \leq k \leq\left(\frac{1}{10}-\varepsilon\right) n$ & $e^{\Omega(n)}$ \\
$(1+1) \mathrm{EA}_{\text {weak }}$ & $m$ constant & $e^{\Omega(n)}$ \\
$(1+1) \mathrm{EA}_{\text {strong }}$ & $m$ constnat & $e^{\Omega(n)}$ \\
$(1+1) \mathrm{EA}_{\varepsilon \text {-constr. }}$ & $m \geq 2$ and constant & $e^{\Omega(n)}$ \\
$\mathrm{SEMO}$ & all & $O\left(k^{2} n \log m\right)$ \\
\hline
\end{tabular}

Table 4. Expected runtimes.

7. J. He and X. Yao. Drift analysis and average time complexity of evolutionary algorithms. Artificial Intelligence, 127:57-85, 2001.

8. M. Laumanns, L. Thiele, and E. Zitzler. Running time analysis of multiobjective evolutionary algorithms on pseudo-boolean functions. IEEE Transactions on Evolutionary Computation, 8(2):170-182, 2004.

9. F. Neumann. Expected runtimes of a simple evolutionary algorithm for the multiobjective minimum spanning tree problem. In Proceedings of the 8th Conference on Parallel Problem Solving from Nature (PPSN '04), volume 3242 of LNCS, pages 80-89. Springer, 2004.

10. T. Storch. On the choice of the population size. In Proc. of the Genetic and Evolutionary Computation Conference (GECCO '04), number 3102 in LNCS, pages 748-760. Springer, 2004.

11. C. Witt. Population size vs. runtime of a simple EA. In Proc. of the 2003 Congress on Evolutionary Computation (CEC '03), volume 3, pages 1996-2003. IEEE Press, 2003.

12. C. Witt. An analysis of the $(\mu+1)$ EA on simple pseudo-boolean functions. In Proc. of the Genetic and Evolutionary Computation Conference (GECCO '04), number 3102 in LNCS, pages 761-773. Springer, 2004. 